\title{
Optimal infusion rate in antimicrobial therapy: explosion of evidence in the last five years
}

This article was published in the following Dove Press journal: Infection and Drug Resistance

\section{Ling-ling Zhu, ' Quan Zhou²}

'VIP care ward, Division of Nursing, The Second Affiliated Hospital, School of Medicine, Zhejiang University, Hangzhou, People's Republic of China; ${ }^{2}$ Department of Pharmacy, the Second Affiliated Hospital, School of Medicine, Zhejiang University, Hangzhou, People's Republic of China

Correspondence: Quan Zhou Department of Pharmacy, the Second Affiliated Hospital, School of Medicine, Zhejiang University, Jiefang Road No 88, Shangcheng District, Hangzhou 310009,

People's Republic of China

Tel +86 57I 87784615

Email zhouquan142602@zju.edu.cn
Background: Sporadic studies in antimicrobial therapy have evaluated the effects of infusion rates on therapeutic and economic outcomes, and new findings may challenge the regular infusion regimen.

Methods: Focusing on studies comparing the outcomes of different infusion regimens, the relevant literature was identified by searching PubMed, Web of Science, and Scopus from January 1, 2013 to March 1, 2018. Papers were finally chosen using a PRISMA flowchart.

Results: Antimicrobials with the superiority of prolonged infusion to standard infusion in terms of efficacy and safety include meropenem, doripenem, imipenem, cefepime, ceftazidime, piperacillin/tazobactam, linezolid, and vancomycin. The strategy of concomitantly reducing total daily dose and prolonging infusion time may cause treatment failure (eg, imipenem). Extended infusion of piperacillin/tazobactam has pharmacoeconomic advantage over standard infusion. Prolonged infusion of voriconazole is inferior to standard infusion because of lower efficacy caused by pharmacokinetic changes. Comparable outcomes following standard infusion and continuous infusion were observed with norvancomycin and nafcillin. Factors determining whether prolonged infusion has a benefit over standard infusion include MIC of bacterial pathogens, bacterial density, diagnosis, disease severity, total daily dose, and renal function.

Conclusion: To maximally preserve the effectiveness of current antimicrobials, effective interventions should be implemented to enhance the application of optimal infusion strategies. For reducing nephrotoxicity, prolonged infusion of meropenem is better than conventional infusion in neonates with Gram-negative late-onset sepsis, and continuous infusion of vancomycin is superior to intermittent infusion. For increasing efficacy, prolonged or continuous infusion of time-dependent antimicrobials (eg, meropenem, doripenem, imipenem, cefepime, ceftazidime, piperacillin/tazobactam, linezolid, and vancomycin) is an optimal choice. Nevertheless, such advantages may only be demonstrated in special clinical circumstances and special populations (eg, patients with a sequential organ failure assessment (SOFA) score $\geq 9$, respiratory tract infections, urinary or intra-abdominal infections, or infections caused by less susceptible pathogens would benefit from prolonged infusion of piperacillin/tazobactam).

Keywords: antimicrobial resistance, antimicrobial stewardship, efficacy, infection, infusion regimen, nursing time, safety, pharmacoeconomics

\section{Introduction}

Infusion rate is one of essential elements that should be included in all intravenous orders or prescriptions according to the Joint Commission International (JCI) Accreditation Standards for Hospitals, sixth edition. ${ }^{1}$ Numerous sporadic clinical studies have been conducted to evaluate the effects of infusion rates on therapeutic and economic 
outcomes of drug therapy, and the new findings have challenged the regular infusion regimen. Health care-associated infections, alongside increasing antimicrobial resistance, pose significant burdens to the hospital system. ${ }^{2}$ More aggressive dosing strategies, such as prolonged and continuous infusion of $\beta$-lactam antibiotics, are applied to address the tremendous challenges associated with the difficult-to-treat pathogens and increase the probability of attaining pharmacokinetic/pharmacodynamic (PK/PD) targets predictive of successful clinical outcomes. Clinicians who have a thorough understanding of appropriate antibiotic use and dosing regimens are essential in the battle to preserve the usefulness of antibiotics and prevent further antibiotic resistance. ${ }^{3,4}$ In this article, based on the literature in the latest 5 years, we share our perspectives in rational intravenous rates of antimicrobial agents and hope to bring some implications for clinical practice and research.

\section{Methods}

\section{Search strategy}

The relevant literature was identified by searching PubMed, Web of Science, and Scopus from January 1, 2013 to March 1, 2018. For PubMed, the search method was identifying papers with titles containing "infusion rate," "infusion rates," "short infusion," "fast infusion," "rapid infusion," "slow infusion," "extended infusion," "prolonged infusion," "continuous infusion," "continuous administration," "infusion time," "infusion mode," "dosing regimen," "dosing regimens," "continuous vs intermittent," "extended vs intermittent," "standard vs prolonged," "extended vs standard," or "intermittent vs prolonged," with a filter of "text availability: abstract" and "languages: English." For Scopus and Web of Science, the query was article title containing the same words as in the PubMed search, with a filter of "language: English; publication type: article," followed by "search within results: antibiotics or antimicrobials."

\section{Selection criteria}

Inclusion criteria included studies comparing the outcomes of different infusion regimens focusing on infusion rates. Exclusion criteria included documents with actually irrelevant topics on antimicrobial therapy, despite being retrieved using the search terms, studies without comparison of outcomes at different intravenous infusion rates of antimicrobial agents, studies lacking a control group with different infusion rates, and documents such as comments, letters, reviews, case reports, editorials, and descriptive studies without statistical analysis. Fifty-three papers were finally chosen according to the inclusion/exclusion criteria. The PRISMA flowchart is illustrated in Figure 1. The full text of each include article was critically reviewed, and valuable information was summarized by data interpretation.

\section{Results and discussion $\beta$-lactam antibiotics}

Two meta-analyses confirmed the advantages of continuous or prolonged infusion of $\beta$-lactam antibiotics. Roberts et $\mathrm{al}^{5}$ performed a meta-analysis of 632 critically ill patients with severe sepsis from three randomized trials, and concluded that administration of $\beta$-lactam antibiotics by continuous infusion was associated with decreased hospital mortality (19.6\% vs $26.3 \%$; relative risk $=0.74 ; P=0.045$ ) and improved clinical cure $(55.4 \%$ vs $46.3 \%$; relative risk $=1.20 ; P=0.021)$ compared with intermittent dosing. Teo et $\mathrm{al}^{6}$ conducted a metaanalysis of 29 studies with 2,206 patients and observed that use of prolonged infusion of $\beta$-lactam antibiotics appeared to be associated with a significant reduction in mortality (relative risk $=0.66$ ) and improvement in clinical success (relative risk=1.12) in hospitalized adult patients compared with intermittent boluses.

Two early studies did not show advantages of continuous or prolonged infusion of $\beta$-lactam antibiotics. A before-after study showed that routine use of prolonged infusion (over 3 hours) for the empiric treatment of Gram-negative bacterial infections in the intensive care unit (ICU) with $\beta$-lactams could not offer advantage over intermittent infusion (over 30 minutes) in terms of treatment success, mortality, or hospital length of stay (LOS). ${ }^{7}$ A multicenter randomized trial showed no difference in outcomes between continuous infusion and 30-minutes intermittent infusion in critically ill patients with severe sepsis receiving $\beta$-lactam antibiotics (eg, ICU-free day, 90-day survival, clinical cure, organ failure-free days, and duration of bacteremia). ${ }^{8}$

A prospective, two-center randomized controlled trial compared clinical and PK/PD outcomes of $\beta$-lactams following continuous infusion or intermittent bolus dosing in adult critically ill patients with severe sepsis not receiving renal replacement therapy. Although two infusion methods did not exhibit a difference in 14 or 30-day survival, continuous infusion resulted in better outcomes in terms of clinical cure rates $(56 \%$ vs $34 \%, P=0.011)$, median ventilator-free days ( 22 vs 14 days, $P<0.043$ ), $\mathrm{PK} / \mathrm{PD}$ target attainment rates at $100 \%$ free drug levels above the minimal inhibitory concentration (fT $>\mathrm{MIC})$ on day $1(97 \%$ vs $70 \%, P<0.001)$ and day $3(97 \%$ vs $68 \%, P<0.001)$ post-randomization compared with intermittent infusion. ${ }^{9}$ 


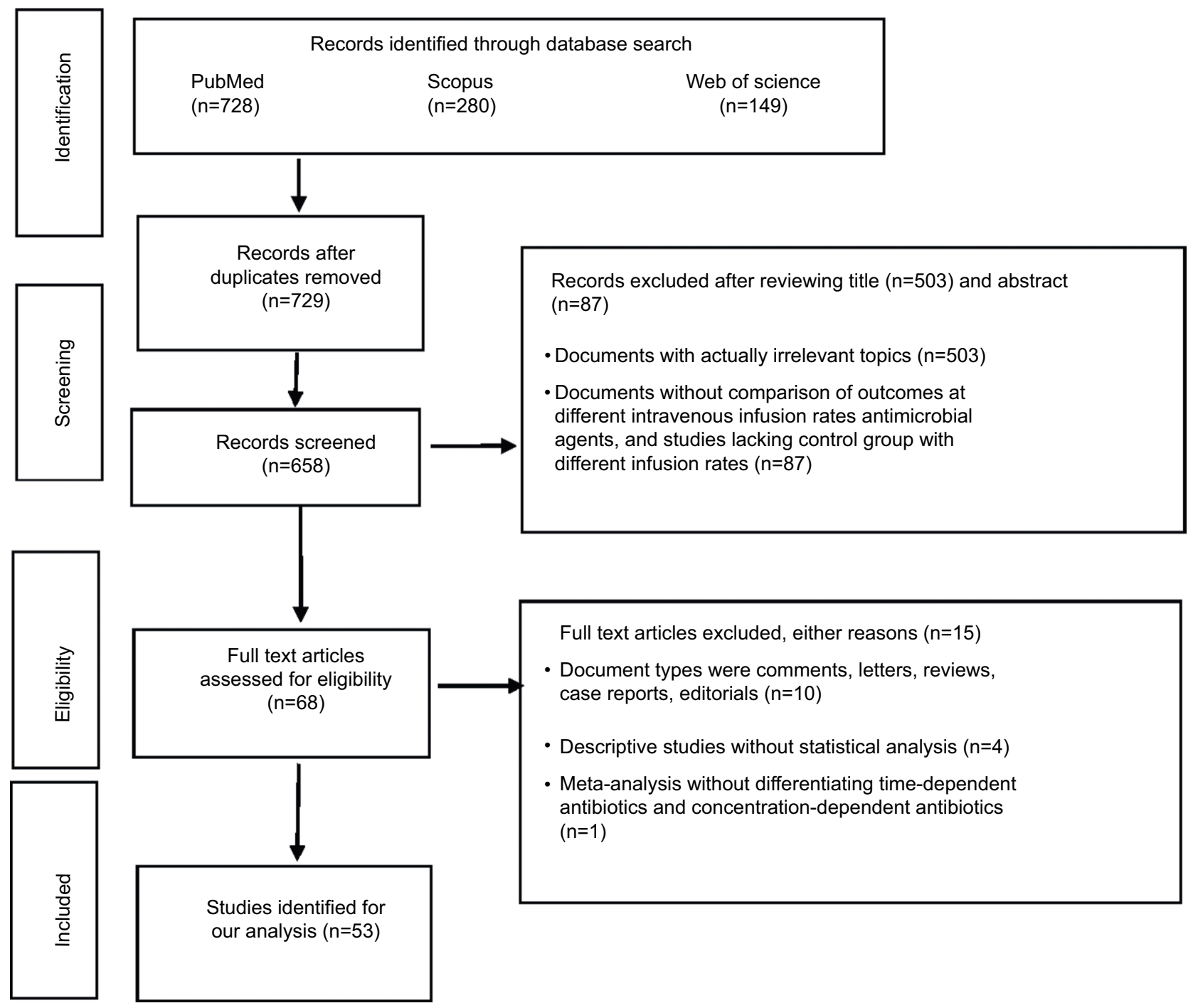

Figure I The flowchart of literature selection.

\section{Meropenem}

There were nine studies of extended or continuous infusion of meropenem vs intermittent administration (Table 1). ${ }^{10-18}$ Among these, two studies were performed in neonates and children, respectively. ${ }^{10,17}$ Six studies were conducted in adults. All studies showed the benefits of prolonged or continuous infusion mode, although they were related with diagnosis, disease severity, and MIC of bacterial pathogens. Only one study revealed the advantage of prolonged infusion mode regarding drug toxicities. ${ }^{10}$

\section{Doripenem}

A retrospective study confirmed that adult critically ill patients receiving standard 1-hour infusion of doripenem for treatment of Gram-negative bacterial infections were significantly more likely to experience clinical failure (odds ratio=5.5) and recurrence of infection or death within 90 days compared with the 4-hour infusion regimen, despite no significant differences in clinical success, LOS, and duration of treatment. ${ }^{19}$

\section{Imipenem}

Ibrahim et $\mathrm{a}^{20}$ compared the efficacy of imipenem in adult ICU patients with ventilator-associated pneumonia (VAP) following regular infusion over 30-60 minutes (first year of the study) or 3-hour extended infusion (second year of the study). Extended infusion resulted in a significant decrease in mortality, number of recurrent infections, ICU LOS, and the number of mechanical ventilator days.

Suchánková et $\mathrm{al}^{21}$ evaluated the population pharmacokinetics of imipenem in adult critically ill patients with hospital-acquired pneumonia (HAP). The 3-hour infusions 
Table I Extended or continuous infusion of meropenem vs intermittent administration

\begin{tabular}{lll}
\hline Study design & Mode of administration and dosage & Pathogen and patients \\
\hline Prospective & Prolonged infusion of meropenem over & Meningitis and \\
randomized & $4 \mathrm{~h}$ vs conventional infusion $(30 \mathrm{~min}) ;$ & Pseudomonas infection in \\
clinical trial & $20 \mathrm{mg} / \mathrm{kg} /$ dose every $8 \mathrm{~h}$ and $40 \mathrm{mg} / \mathrm{kg} /$ & neonates with Gram- \\
& dose every $8 \mathrm{~h}$ & negative late-onset sepsis
\end{tabular}

Retrospective observational study

Randomized clinical trial

\section{Observational} study using a population PK/ PD model Comparative study using an in vitro $P K / P D$ model

Observational pharmacokinetic study using Monte Carlo simulations

Prospective, multicenter pharmacokinetic point-prevalence study with a post hoc analysis
$4 \mathrm{~h}$ extended infusion of meropenem ( I g/8 h) vs conventional 30 min short infusion at the same dose

Continuous group: a loading dose of $0.5 \mathrm{~g}$ of meropenem infused over 30 min followed by continuous infusion of $3 \mathrm{~g} /$ day, which was divided into six consecutive $4 \mathrm{~h}$ continuous infusions of meropenem $0.5 \mathrm{~g}$; intermittent group: an initial dose of $1.5 \mathrm{~g}$ followed by $\mathrm{I} \mathrm{g}$ infused over 30 min every $8 \mathrm{~h}$. $3 \mathrm{~h}$ and $30 \mathrm{~min}$ infusion regimens at a dose of meropenem I $g$ (twice or thrice daily)

Short $(0.5 \mathrm{~h})$ and prolonged $(3 \mathrm{~h})$ infusion regimens of $\mathrm{I} g$ meropenem every $8 \mathrm{~h}$

Meropenem as sole agent or in combination with other antimicrobials

Intermittent-bolus administration vs prolonged infusion
Adult patients with febrile neutropenia

Adult patients with severe sepsis and septic shock

Adult critically ill patients

Different adult patient groups with CPKP isolates

Acinetobacter baumannii and Pseudomonas aeruginosa infections in adult patients with septic shock

Critically ill patients
Outcomes

Higher clinical improvement (61\% vs 33\%,

$P=0.009)$ and microbiologic eradication at the 7 th day of meropenem therapy ( $82 \%$ vs $56.8 \%$, $P=0.009)$, lower neonatal mortality ( $14 \%$ vs $31 \%$, $P=0.03)$, shorter duration of respiratory support ( 12.5 days vs 4 days, $P=0.03$ ), and less acute kidney injury ( $6 \%$ vs $23.5 \%, P=0.02) .{ }^{10}$

Better clinical outcome (ie, fewer additional antibiotics during the first 5 days of treatment, a more prompt defervescence and a faster decrease in $C$-reactive protein level) $(P<0.05)$ despite comparable LOS in the hospital and mortality rate between two groups."

Continuous infusion of meropenem provided significantly shorter treatment duration (7.6 vs 9.4 days; $P=0.035$ ) while clinical success was similar between both groups. For mediumsusceptibility pathogens, concentrations above the MIC in the continuous infusion group were $100 \%$, which was better than that in the intermittent group. ${ }^{12}$

The PTA, T>MIC of $100 \%$, was higher for $3 \mathrm{~h}$ infusion regimen compared with 30 min infusion regimen for all ranges of creatinine clearance. ${ }^{13}$

ICU patients exhibited the lowest target attainment rates, whereas internal medicine patients achieved the highest target attainment rates. ${ }^{14}$ The PTA (ie, 40\% T>MIC) with short infusion was higher than those with prolonged infusion for isolates with MIC 4-8 $\mathrm{mg} / \mathrm{L}$ rather than $\mathrm{MIC} \geq 16 \mathrm{mg} / \mathrm{L}$ or $\leq 2 \mathrm{mg} / \mathrm{L}$ (MIC $4 \mathrm{mg} / \mathrm{L}$ : 98\%-99\% vs 6I\%-83\%; MIC 8 mg/L: $55 \%-79 \%$ vs $23 \%-33 \%) .{ }^{14}$

Intermittent dosing of I g/8 h (30 min) seemed sufficient in patients with normal renal function, whereas increasing doses $(2 \mathrm{~g} / 6 \mathrm{~h}$ for A. baumannii, $2 \mathrm{~g} / 8 \mathrm{~h}$ or I g/6 h for $P$. aeruginosa) by intermittent $(30 \mathrm{~min})$ or prolonged $(3 \mathrm{~h})$ infusion or continuous infusion $(6 \mathrm{~g}$ over 24 h) could increase the possibility of achieving therapeutic drug concentrations in patients with septic shock and possible augmented renal clearance. $^{15}$

Compared with intermittent-bolus administration, prolonged infusion demonstrated significantly better 30-day survival in the subgroup of patients with respiratory infection (86.2\% vs $56.7 \% ; P=0.012$ ), and higher clinical cure rate $(73.3 \%$ vs $35.0 \% ; P=0.035)$ and survival rates $(73.3 \%$ vs $25.0 \%$; $P=0.025)$ in patients with a SOFA score of $\geq 9$. However, two infusion methods did not exhibit a significant difference in 30-day survival rate in patients receiving antimicrobial treatment and in the subgroup of patients with abdominal infection. ${ }^{16}$ 
Table I (Continued)

\begin{tabular}{|c|c|c|c|}
\hline Study design & Mode of administration and dosage & Pathogen and patients & Outcomes \\
\hline $\begin{array}{l}\text { Multicenter study } \\
\text { using population } \\
\text { pharmacokinetics } \\
\text { analysis }\end{array}$ & $\begin{array}{l}\text { Prolonged } 3 \mathrm{~h} \text { infusion vs } 30 \mathrm{~min} \\
\text { infusion of meropenem } 40 \mathrm{mg} / \mathrm{kg} \text { every } \\
8 \mathrm{~h}\end{array}$ & $\begin{array}{l}\text { Children with cystic } \\
\text { fibrosis }\end{array}$ & $\begin{array}{l}\text { At MICs of I, } 2 \text {, and } 4 \mathrm{mg} / \mathrm{L}, \text { PTAs for the } \\
0.5 \mathrm{~h} \text { infusion were } 87.6 \%, 70.1 \% \text {, and } 35.4 \% \text {, } \\
\text { respectively. Prolonged infusion increased PTAs } \\
\text { to }>99 \% \text { for these MICs and achieved } 82.8 \% \text { at } \\
8 \mathrm{mg} / \mathrm{L} \text {, ie, } 3 \mathrm{~h} \text { infusion provided an exposure } \\
\text { benefit against pathogens with MICs } \geq 1 \mathrm{mg} / \mathrm{L} .{ }^{17}\end{array}$ \\
\hline $\begin{array}{l}\text { Randomized } \\
\text { controlled trial }\end{array}$ & $\begin{array}{l}\text { Meropenem administered as a I g, } 30 \\
\text { min infusion or as a } 500 \mathrm{mg}, 3 \mathrm{~h} \text { infusion }\end{array}$ & Critically ill patients & $\begin{array}{l}\text { Meropenem I g infused over } 30 \text { min could } \\
\text { achieve a similar } \% \mathrm{~T}>\mathrm{MIC} \text { to meropenem } 500 \\
\mathrm{mg} \text { given over } 3 \mathrm{~h} \text { in critically ill patients. For } \\
\text { low MICs }(\leq 2 \mathrm{mg} / \mathrm{L}) \text {, both regimens attained } \\
\text { a } \% \mathrm{~T}>\mathrm{MIC}>40 \% \text { in all patients. For an } \mathrm{MIC} \text { of } \\
4 \mathrm{mg} / \mathrm{L} \text {, this target was attained in all but one } \\
\text { patient; however, with an MIC of } 8 \mathrm{mg} / \mathrm{L} \text {, three } \\
\text { patients in each group had a } \% \mathrm{~T}>\mathrm{MIC}<40 \% \text {. For } \\
\text { MICs up to } 8 \mathrm{mg} / \mathrm{L} \text {, the two regimens exhibited } \\
\text { no difference in target attainment. }{ }^{18}\end{array}$ \\
\hline
\end{tabular}

Abbreviations: CPKP, carbapenemase-producing Klebsiella pneumoniae; h, hours; ICU, intensive care unit; LOS, length of stay; MIC, minimum inhibition concentration; min, minutes; PK/PD, pharmacokinetic/pharmacodynamic; PTA, probability of target attainment; SOFA, sequential organ failure assessment; T>MIC, time for which drug concentrations exceed the MIC.

and continuous infusion achieved over $97 \%$ of cumulative fraction of response (CFR) against all pathogens, with the exception of Pseudomonas aeruginosa and Acinetobacter spp., where the maximum CFRs were $85.5 \%$ and $88.4 \%$, respectively. The 3-hour extended infusions and continuous infusion achieved higher probability of target attainment (PTA) for the targets of achieving fT $>$ MIC for $40 \%$ and $100 \%$ of the dosing interval as PK/PD targets compared with 0.5 hour infusions; however, continuous infusion carried a risk of not reaching the MIC of less susceptible pathogens in a higher proportion of patients. Therefore, it may be necessary to use maximum doses administered as extended infusions in critically ill patients with HAP and risk factors for Gramnegative non-fermenting bacteria.

The benefit of prolonged infusion vs standard infusion of imipenem may be related with drug dose and MIC of bacterial pathogens. Lipš et $\mathrm{al}^{22}$ compared the $\mathrm{PK} / \mathrm{PD}$ indices of imipenem administered by standard infusion ( $1 \mathrm{~g}$ imipenem $/ 1$ g cilastatin over 30 minutes every 8 hours) and extended infusion with a reduced total dose $(0.5 \mathrm{~g}$ imipenem $/ 0.5 \mathrm{~g}$ cilastatin over 3 hours every 6 hours) in critically ill patients with nosocomial pneumonia. Concentrations of imipenem were above the target concentration $(4 \times \mathrm{MIC}$ of $2 \mathrm{mg} / \mathrm{L})$ for $\geq 40 \%$ of the dosing interval in every patient in the bolus group, whereas in the extended group this $\mathrm{PK} / \mathrm{PD}$ index ( $40 \% \mathrm{fT}>4 \times \mathrm{MIC}$ ) was achieved in only $20 \%$ of patients. Moreover, $70 \%$ of the patients in the extended group did not reach the desired drug concentrations at all. For a MIC of
$4 \mathrm{mg} / \mathrm{L}$, only one patient in the bolus group achieved $40 \%$ $\mathrm{fT}>4 \times \mathrm{MIC}$, and the rest of the patients still at least reached concentrations above $4 \times \mathrm{MIC}$, whereas none of the patients in the extended group reached these concentrations. Therefore, administration of $0.5 \mathrm{~g}$ of imipenem by a 3-hour infusion every 6 hours could not provide adequate drug concentrations to control infections caused by pathogens with $\mathrm{MIC} \geq 2$ $\mathrm{mg} / \mathrm{L}$. In empirical treatment where the presence of a less sensitive micro-organism cannot be excluded, the strategy of concomitantly reducing total daily dose and prolonging infusion time may cause treatment failure.

\section{Biapenem}

The usual biapenem infusion regimen $(0.3$ g every 12 hours, 0.5 -hour infusion) may result in unsatisfactory clinical outcomes in Chinese adult patients with lower respiratory tract infections, because PTA could not achieve $\geq 90 \%$ with an MIC of $4 \mu \mathrm{g} / \mathrm{mL}$. With prolonging infusion time of biapenem from 0.5 hour to 3 hours with a dose of $0.3 \mathrm{~g}$ every 6 hours, the PTAs for achieving 40\% T $>$ MIC with an MIC of $4 \mu \mathrm{g} / \mathrm{mL}, 60 \% \mathrm{~T}>\mathrm{MIC}$ with an MIC of $2 \mu \mathrm{g} / \mathrm{mL}$, and $80 \% \mathrm{~T}>\mathrm{MIC}$ with an MIC of $1 \mu \mathrm{g} / \mathrm{mL}$ were increased from $53.8 \%$ to $94.3 \%$, from $60.3 \%$ to $98.4 \%$, and from $62.8 \%$ to $96.2 \%$, respectively. Higher doses and longer infusion time of biapenem would be appropriate for empirical therapy, and combination therapy with other antibacterials may be more appropriate if $P$. aeruginosa or Acinetobacter baumannii infection is suspected. ${ }^{23}$ 


\section{Ertapenem}

Prescribing information for ertapenem specifies that the drug should be infused over a period of 30 minutes when administered intravenously. Wiskirchen et a ${ }^{24}$ showed that ertapenem $1 \mathrm{~g}$ administered as a rapid 5-minute infusion could provide a well-tolerated, bioequivalent, and pharmacodynamically equivalent regimen to the 30-minute infusion at clinically relevant MICs in healthy adult volunteers. The estimated PTA was greater than $90 \%$ when using a bactericidal target of $40 \% \mathrm{fT}>\mathrm{MIC}$ up to an MIC of $0.25 \mathrm{mg} / \mathrm{L}$ for both infusion times. Additional studies in infected patients are needed to determine the safety and value of rapid 5-minute infusion of ertapenem.

\section{Cefepime}

A retrospective quasi-experimental study showed that the extended infusion (a 4-hour infusion of $2 \mathrm{~g}$ cefepime every 8 hours) rather than standard infusion (a 30-minute infusion of $2 \mathrm{~g}$ cefepime every 8 hours) resulted in significantly lower overall mortality, reduced hospital costs, and significantly shorter LOS in the treatment of $P$. aeruginosa infections in adult ICU patients. ${ }^{25}$ However, a newer study did not confirm the superiority of extended infusion of cefepime. ${ }^{26}$ Wrenn et $\mathrm{a}^{26}$ compared the outcomes between extended infusion and standard infusion of cefepime as empiric treatment of febrile neutropenia in adult patients admitted to a hematology/oncology service and observed no statistically significant differences in defervescence by 72 hours and secondary outcome measures (time to defervescence, clinical success, in-hospital mortality, hospital LOS, and need for additional antimicrobials).

Regarding continuous infusion of cefepime, this infusion mode could significantly enhance the antibacterial effect toward intracranial infections. Compared to intermittent infusion of $2 \mathrm{~g}$ cefepime every 12 hours, continuous infusion of $4 \mathrm{~g} /$ day cefepime over 24 hours could significantly reduce the treatment duration in adult neurosurgical patients with postoperative intracranial infections ( $6.6 \pm 1.9$ days vs $7.8 \pm 2.6$ days; $P=0.036$ ), and achieve higher $\% \mathrm{~T}>\mathrm{MIC}$ for cefepime concentrations in cerebrospinal fluid (for MICs of $4 \mu \mathrm{g} / \mathrm{mL}$, $83.3 \%$ vs $25 \%$; for MICs of $8 \mu \mathrm{g} / \mathrm{mL}, 75 \%$ vs $0 \%$ ). ${ }^{27}$

\section{Ceftazidime}

Cousson et $\mathrm{al}^{28}$ confirmed the advantages of continuous infusion (loading dose of $20 \mathrm{mg} / \mathrm{kg}$ followed by $60 \mathrm{mg} / \mathrm{kg} / \mathrm{day}$ ) over intermittent infusion $(20 \mathrm{mg} / \mathrm{kg}$ over 30 minutes every 8 hours) in terms of pharmacodynamics and predictable efficacy in adult patients with VAP due to Gram-negative bacilli. The $\% \mathrm{~T}>\mathrm{MIC}$ of ceftazidime (MIC=20 mg/L) was $100 \%$ and $46 \%$, respectively, following continuous infusion and intermittent infusion $(P<0.003)$.

\section{Piperacillin/tazobactam Efficacy issue}

Compared to the conventional intermittent dosage regimen, prolonged piperacillin/tazobactam infusion resulted in shorter ICU and hospital LOS among adult critically ill patients (ie, saving 0.6 days and 5.6 days, respectively) ${ }^{29}$ a significantly lower rate of readmission within 30 days of hospital discharge $(1.2 \%$ vs $13.7 \%, P=0.002),{ }^{30}$ and greater PTA in critically ill patients receiving continuous renal replacement therapy or critically ill patients with severe sepsis or septic shock undergoing continuous venovenous hemofiltration. ${ }^{31,32}$

\section{Safety issues}

There were two studies specially comparing safety issues of extended infusion vs intermittent infusion of piperacillin/ tazobactam. Both intermittent and extended-infusion groups experienced similar incidence of acute renal injury in hospitalized patients, ${ }^{33}$ and comparable nephrotoxicity in patients with a creatinine clearance $(\mathrm{CrCl}) \geq 40 \mathrm{~mL} / \mathrm{min}$ receiving at least 96-hour combination therapy of vancomycin and piperacillin/tazobactam. ${ }^{34}$

\section{Pharmacoeconomic issues}

There were four studies involving the pharmacoeconomic issues of extended infusion vs intermittent infusion of piperacillin/tazobactam. Brunetti et $\mathrm{al}^{35}$ showed that automatic substitution of extended infusion for standard infusion of piperacillin/tazobactam in adult patients could reduce total cost per treatment course by $13 \%$, with no difference in LOS and inhospital mortality between two groups. Compared with regular infusion in the pre-intervention phase, post-implementation of an extended infusion dosing protocol resulted in a significant shorter ICU and hospital LOS and a significantly reduced cost of therapy ( $\$ 120.21$ vs $\$ 155.17 ; P=0.035$ ), although there was no significant difference in the primary end-point of mortality and adverse drug effects between the two phases. ${ }^{36}$ Bao et al ${ }^{37}$ showed better $\mathrm{PK} / \mathrm{PD}$ and pharmacoeconomic parameters following 3-hour extended infusion of piperacillin/tazobactam vs 30-minute intermittent infusion every 6 hours in the treatment of HAP in adult critically ill patients with low illness severity. Extended infusion achieved a lower therapy cost $(\$ 1,351.72$ vs $\$ 1,782.04, P=0.001)$, and higher $\% \mathrm{fT}>\operatorname{MIC}(\sim 100,98.73$, and $93.04 \%$ in the extended infusion group vs $81.48,53.29$, and $42.15 \%$ in the intermittent infusion group, respectively, 
for the pathogen with an MIC of 4, 8, and $16 \mathrm{mg} / \mathrm{L}$ ), although the clinical success rate, clinical failure rate, and drug-related adverse events did not significantly differ between groups. Cotrina-Luque et $\mathrm{al}^{38}$ confirmed similar efficacy and safety of continuous infusion of piperacillin/tazobactam to intermittent infusion in treating $P$. aeruginosa infection; however, continuous infusion (an initial loading dose of piperacillin/tazobac$\operatorname{tam} 2 / 0.25 \mathrm{~g}$ in $100 \mathrm{~mL}$ of saline solution for 30 minutes, immediately followed by continuous infusion of piperacillin/ tazobactam $8 / 1 \mathrm{~g}$ in $500 \mathrm{~mL}$ of saline over 24 hours) could lead to system cost savings due to utilizing a 30\% lower dose of piperacillin/tazobactam than intermittent infusion (an initial dose of piperacillin/tazobactam 4/0.5 $\mathrm{g}$ in $100 \mathrm{~mL}$ of saline solution for 30 minutes, followed by piperacillin/tazobactam $4 / 0.5 \mathrm{~g}$ in $100 \mathrm{~mL}$ of saline for 30 minutes every 8 hours).

\section{Whether prolonged infusion has a benefit over standard infusion depends on the MICs of bacterial pathogens}

Zelenitsky et $\mathrm{al}^{39}$ characterized the pharmacodynamics of prolonged-infusion piperacillin/tazobactam in an in vitro pharmacodynamic model of $P$. aeruginosa. The benefits of prolonged infusion were selective and most likely observed in patients with less susceptible pathogens, ie, prolonged infusion had no advantages over standard infusion against isolates with susceptible MICs of 8 or $16 \mathrm{mg} / \mathrm{L}$, whereas it produced more than twice the final bacterial kill against less susceptible isolates with an intermediate MIC of $32 \mathrm{mg} / \mathrm{L}$.

Nichols et $\mathrm{al}^{40}$ evaluated the population $\mathrm{PK} / \mathrm{PD}$ profiles for dosing regimens of $80-100 \mathrm{mg} / \mathrm{kg}$ of the piperacillin component given every 6-8 hours and infused over $0.5,3$, or 4 hours in critically ill children. At the pharmacodynamic target of a $\mathrm{T}>\mathrm{MIC}$ of $\geq 50 \%$, all simulated dosing regimens achieved a PTA of $>90 \%$ at MICs of $\leq 8 \mathrm{mg} / \mathrm{L}$. Only 0.5 -hour infusion regimens of $80-100 \mathrm{mg} / \mathrm{kg}$ every 8 hours did not achieve a PTA of $>90 \%$ at a MIC of $16 \mathrm{mg} / \mathrm{L}$. All extendedinfusion regimens achieved PTAs of $>90 \%$ at MICs of $\leq 16$ $\mathrm{mg} / \mathrm{L}$; however, only the 3-hour infusion regimens given every 6 hours achieved PTAs of $>90 \%$ at a MIC of $32 \mathrm{mg} / \mathrm{L}$. At the pharmacodynamic target of a $\mathrm{T}>\mathrm{MIC}$ of $100 \%$, none of the regimens achieved a PTA of $>90 \%$ at a MIC of $\geq 16$ $\mathrm{mg} / \mathrm{L}$, and only $100 \mathrm{mg} / \mathrm{kg}$ given every 6 hours and infused over 3 hours achieved a PTA of $>90 \%$ at a MIC of $8 \mathrm{mg} / \mathrm{L}$. For pathogens with MICs of $\leq 8 \mathrm{mg} / \mathrm{L}$, extended infusion did not substantially improve PTA, and standard infusion was likely sufficient. However, piperacillin/tazobactam 100/12.5 $\mathrm{mg} / \mathrm{kg}$ given as an extended infusion every 6-8 hour may be optimal for empirical or directed therapy in critically ill pediatric patients with infections caused by less susceptible pathogens or if the desirable $\mathrm{T}>\mathrm{MIC}$ is greater than $50 \%$.

\section{Whether prolonged infusion has a benefit over standard infusion depends on the diagnosis and disease severity}

Lyu et $\mathrm{al}^{41}$ investigated the infusion modes of piperacillin/ tazobactam guided by SOFA score in cancer patients with postoperative HAP. Compared to conventional infusion (30 minutes), prolonged infusion improved patient survival, increased clinical efficacy and bacteriologic efficacy, and reduced 28-day mortality $(P<0.05)$. However, patients with severe disease (SOFA score $\geq 9$ ), rather than those with mild disease (SOFA score $<9$ ), benefited from prolonged infusion (eg, shorter days of antibiotics use and ventilator time, longer survival, better clinical efficacy and lower 28-day mortality rate).

Extended infusion of piperacillin/tazobactam over 4 hours for presumed sepsis syndromes exhibited a shorter duration of piperacillin/tazobactam therapy than regular infusion over 30 minutes $(P<0.001)$, with no significant difference in inpatient mortality rates, overall LOS and clinical failure rates between two infusion methods. Subgroup analysis revealed that ICU patients exhibited no significant differences in outcomes following two dosing methods, whereas patients with urinary or intra-abdominal infections had lower mortality and clinical failure rates following extended-infusion treatment. ${ }^{42}$

Critically ill patients who had a diagnosis of either bacterial infection or neutropenic fever receiving a 4-hour extended-infusion piperacillin/tazobactam demonstrated similar 14-day mortality compared with patients receiving 30-minute intermittent infusions. However, post hoc subgroup analysis showed a lower mortality rate in the extendedinfusion group who had infectious organisms identified $(9.3 \%$ vs $22.4 \%, P=0.01$ ) or were diagnosed with respiratory tract infections ( $8.9 \%$ vs $18.7 \%, P=0.02) .{ }^{43}$

\section{Whether prolonged infusion has a benefit over standard infusion depends on bacterial density}

Bacterial burden may exert a significant influence on the ultimate outcome of antimicrobial therapy. Felton et a $1^{44}$ used a hollow-fiber infection model with P. aeruginosa to examine the impact of administering piperacillin/tazobactam by 0.5 hour bolus dosing or 4 hours extended infusion every 8 hours on the emergence of piperacillin resistance. Both infusion methods resulted in comparable antibacterial activities and rates of emergence of antimicrobial resistance. For the lower initial bacterial density, trough total plasma piperacillin 
concentration/MIC ratios of 3.4 and 10.4 following bolus and extended-infusion regimens, respectively, were able to suppress the emergence of piperacillin resistance. For the higher initial bacterial density, both infusion regimens were associated with progressive growth of a resistant subpopulation, and thus combination therapy may be required to maximize bacterial killing and prevent antimicrobial resistance.

\section{Nafcillin}

Prescribing information for nafcillin specifies that the drug should be administered slowly as an intravenous infusion over at least 30-60 minutes at the adult dose of $500 \mathrm{mg}$ to 1 g every 4 hours. Bauer et $\mathrm{al}^{45}$ compared the clinical efficacy and incidence of acute interstitial nephritis or acute renal failure between nafcillin intermittent ( 2 g every 4 hours for 30 minutes) vs continuous (6 g every 12 hours twice daily) infusion in patients with methicillin-sensitive Staphylococcus aureus bacteremia. Results showed no difference in the safety and efficacy between two infusion methods.

\section{Linezolid}

Linezolid injection (Zyvox ${ }^{\circledR}$; Pfizer Inc., New York, NY, USA) should be administered by intravenous infusion over a period of 30-120 minutes. A clinical trial showed that 2-hour infusion of linezolid (600 mg) was the most effective regimen among three different infusion regimens. The PTA of the 4-hour infusion regimen was much lower compared with the 0.5 and 2-hour infusion regimens in terms of the ratio of the area under the curve to the MIC (AUC/MIC), whereas the PTA of 2-hour and 4-hour regimens rather than the 0.5-hour regimen remained $100 \%$ in terms of $\% \mathrm{~T}>\mathrm{MIC} .{ }^{46}$

de Pascale et $\mathrm{al}^{47}$ compared linezolid concentrations in plasma and epithelial lining fluid following administration in obese critically ill patients with VAP by 60 -minute intermittent (600 mg every 12 hours) and continuous infusion (600 mg intravenous loading dose infused over 60 minutes, followed by $1,200 \mathrm{mg}$ continuous infusion over 24 hours). Continuous infusion resulted in a significantly longer median time of linezolid plasma concentration persisted above MIC ranging from $1-4 \mathrm{mg} / \mathrm{L}$ and a higher epithelial lining fluid/ plasma penetration ratio $(98.8 \%$ vs $87.1 \%$; $P<0.001)$ compared with intermittent infusion.

\section{Glycopeptide antibiotics} Vancomycin

Tafelski et $\mathrm{al}^{48}$ compared the effects of different dosing regimens on vancomycin target levels in critically ill patients. Intermittent administration in adults was as follows: $500 \mathrm{mg}$ vancomycin every 6 hours given over 1 hour or 1 g every 12 hours given over 1 hour. The continuous infusion regimen was as follows: initiation of therapy with $1 \mathrm{~g}$ vancomycin as an infusion given over 1 hour, followed by 24 hour-continuous infusion of $1 \mathrm{~g}$ vancomycin in $50 \mathrm{~mL}$ of solution at different infusion rates (normal renal function: $4.2 \mathrm{~mL} / \mathrm{h}$; impaired renal function and $\mathrm{CrCl}$ of $<50 \mathrm{~mL} / \mathrm{min}$ : $2.1 \mathrm{~mL} / \mathrm{h}$; impaired renal function and $\mathrm{CrCl}$ of $<20 \mathrm{~mL} / \mathrm{min}$ : $1 \mathrm{~mL} / \mathrm{h}$ ). Patients with continuous administration achieved target serum levels significantly earlier and showed fewer sub-therapeutic serum levels $(41 \%$ vs $11 \%, P<0.001)$.

Compared to intermittent dosing, continuous infusion of vancomycin could result in the following outcomes: (1) higher target attainment rate for therapeutic vancomycin exposure ( $48 \%$ vs $19 \%, P<0.001)$ and less between-patient variation in vancomycin serum level in critically ill patients; ${ }^{49}$ (2) greater ( $40 \%$ vs $21.5 \%, P=0.02)$ and more rapid achievement of goal plasma concentrations (2.04 vs 3.76 days, $P<0.0001)$, higher pharmacodynamic target attainment (92.3\% vs $30.8 \%, P<0.0001)$, and less dose adjustments, days of therapy, and mean total daily dose requirements per patient without significant difference in nephrotoxicity in neurosurgical ICU patients; ${ }^{50}$ (3) more quickly achieving the target level, greater percentage of keeping therapeutic 24-hour levels ( $100 \%$ vs $4.4 \%, P<0.001)$, and higher mean 24-hour vancomycin levels $(20.35 \pm 2.78 \mu \mathrm{g} / \mathrm{mL}$ vs $9.7 \pm 3.52$ $\mu \mathrm{g} / \mathrm{mL}, P<0.001)$ in adult ICU patients during continuous venovenous hemofiltration; ${ }^{51}$ (4) higher serum vancomycin level $(19.8 \pm 6.13 \mu \mathrm{g} / \mathrm{mL}$ vs $8.9 \pm 3.9 \mu \mathrm{g} / \mathrm{mL}, P<0.0001)$ and percentage of keeping drug level in the therapeutic range $(57.1 \%$ vs $7.4 \%, P<0.0001)$ in trauma patients with suspected VAP. To avoid inadequate vancomycin level, continuous vancomycin infusion should be the optimal dosing strategy. ${ }^{52}$

Hanrahan et $\mathrm{al}^{53}$ conducted a meta-analysis of seven studies of vancomycin administration by continuous vs intermittent infusion and identified a non-significant trend of reduced nephrotoxicity in those who received vancomycin by continuous infusion (risk ratio $=0.799, P=0.299$ ). However, a newer meta-analysis of eleven studies by Hao et $\mathrm{al}^{54}$ revealed that patients treated with continuous infusion of vancomycin had a significantly lower incidence of nephrotoxicity compared with patients receiving intermittent infusion (relative risk $=0.61$, $P<0.001$ ), whereas the clinical efficacy was not significantly different.

\section{Norvancomycin}

$\mathrm{Wu}$ et $\mathrm{al}^{55}$ failed to observe significant advantages of a continuous infusion method of norvancomycin (ie, $0.8 \mathrm{~g}$ norvancomycin for 1 hour, and then another $0.4 \mathrm{~g}$ for 11 hours with 
extended infusion, followed by continuous infusion of $0.4 \mathrm{~g}$ for 12 hours) over the conventional infusion method (ie, 0.8 g norvancomycin for 1 hour every 12 hours) in an early stage after neurosurgery. The cerebral spinal fluid concentration in both groups reached or exceeded the MIC90 (2 mg/L) of target bacteria such as methicillin-resistant Staphylococcus aureus (MRSA). This pilot study of norvancomycin in a neurosurgical individual needs to be confirmed by further large-scale studies.

\section{Fosfomycin}

Albiero et $\mathrm{al}^{56}$ evaluated treatment regimens of the meropenem-fosfomycin combination against carbapenemaseproducing Klebsiella pneumoniae (CPKP). For the 0.5 -hour infusion, fosfomycin monotherapy regimens did not achieve a $90 \%$ PTA of $>70 \%$ fT $>$ MIC against the MIC50 or MIC90 in patients with normal renal function. Prolonged infusion of fosfomycin over 3 hours improved the PTA over that of a 0.5-hour infusion, however, fosfomycin monotherapy regimens did not reach 90\% PTA against either the MIC50 or the MIC90 in patients with normal renal function. In combination therapy of meropenem, fosfomycin $6 \mathrm{~g}$ every 6 hours or 8 g every 8 hours as a 3-hour infusion had better chances of achieving PTA.

\section{Voriconazole}

Prescribing information for voriconazole (Vfend ${ }^{\circledR}$; Pfizer Inc.) requires a maximum infusion rate of $3 \mathrm{mg} / \mathrm{kg}$ per hour over 1-2 hours. Hohmann et $\mathrm{al}^{57}$ observed that infusion rate could modulate voriconazole pharmacokinetics. Maximum concentration after a 4-hour infusion, and after a 6-hour infusion was $29 \%$ and $51 \%$ lower compared to a $400 \mathrm{mg} / 2$-hour infusion, and fell outside of the bioequivalence margins (0.80-1.25). The AUC of $400 \mathrm{mg}$ intravenous voriconazole was $16 \%$ lower when administered over 6 hours compared to 2 hours infusion. Metabolic clearance of CYP3A probe midazolam was $516 \mathrm{~mL} /$ min following $100 \mathrm{mg} / 4$ hours voriconazole, $152 \mathrm{~mL} / \mathrm{min}$ for $400 \mathrm{mg} / 6$ hours, $192 \mathrm{~mL} / \mathrm{min}$ for $400 \mathrm{mg} / 4$ hours, and $202 \mathrm{~mL} /$ $\mathrm{min}$ for $400 \mathrm{mg} / 2$ hours, which reflected an auto-inhibitory effect on CYP3A4-mediated voriconazole metabolism. Voriconazole efficacy correlates well in vivo with the AUC/MIC or mean unbound voriconazole concentration/MIC ratio. ${ }^{58}$ Thus, to avoid reduced exposure and potential efficacy, infusion rate of voriconazole should not deliberately be prolonged.

\section{Practical issues in the choice of infusion rate}

When implementing the prolonged or continuous infusion of antimicrobial therapy, several pragmatic issues should be considered, including availability of intravenous catheters, compatibility of intravenous drugs, drug stability, safety concerns, monitoring for efficacy and toxicity, and timing of administration.

\section{Compatibility}

Critically ill patients often receive multiple drugs via continuous intravenous infusion. Intravenous drugs may be infused concurrently via Y-site when compatibility data are available. For example, vancomycin was stable during the whole process and also during 72 hours exposure of concentrated solutions at temperatures up to $37^{\circ} \mathrm{C}$, but careful attention must be paid to incompatibilities with $\beta$-lactams (temocillin, piperacillin/tazobactam, ceftazidime, imipenem, cefepime, and flucloxacillin), moxifloxacin, propofol, valproic acid, phenytoin, theophylline, methylprednisolone, and furosemide. ${ }^{59}$ For combination therapy of cefepime and vancomycin, worries about incompatibility between two antibiotics would result in conventional practice (ie, flush the tube with diluent and initiate vancomycin infusion when cefepime infusion over 30-60 minutes is completed). Tran et $\mathrm{al}^{60}$ evaluated whether an intravenous push of cefepime (a mixture of $10 \mathrm{~mL}$ normal saline injected over 2-5 minutes) was associated with a shorter time to vancomycin administration in the emergency department compared with intravenous piggyback infusion (a mixture of $100 \mathrm{~mL}$ normal saline infused over 30-60 minutes). The transition from intravenous piggyback infusion to intravenous push of cefepime could statistically significantly decrease the time to initiation of vancomycin administration by more than 1 hour to patients with suspected infections who were ordered both antibiotics. To prevent delay to MRSA coverage with vancomycin in the emergency department, it is reasonable to administer the first dose of cefepime as an intravenous push.

Meropenem/vaborbactam is administered as a 3-hour infusion given every 8 hours, thereby potentially restricting an intravenous line for 9 hours/day. Of the 88 medications tested, meropenem/vaborbactam was compatible with 73 (83\%), including antibiotics such as aminoglycosides, colistin, fosfomycin, linezolid, tigecycline, and vancomycin. Physical incompatibility was observed with albumin, amiodarone, anidulafungin, calcium chloride, ceftaroline, ciprofloxacin, daptomycin, dobutamine, isavuconazole, midazolam, nicardipine, ondansetron, and phenytoin. ${ }^{61}$

\section{Stability}

Continuous infusion of meropenem may be applied in clinical settings at normal and elevated temperatures. The $5 \mathrm{mg} / \mathrm{mL}$ 
aqueous reconstituted solution of meropenem was stable for up to 8 hours in the temperature range between $25^{\circ} \mathrm{C}$ and $35^{\circ} \mathrm{C}$, and for up to 5 hours at $40^{\circ} \mathrm{C}$. ${ }^{62}$ The $40 \mathrm{mg} / \mathrm{mL}$ solution of meropenem reconstituted in isotonic saline was stable at $25^{\circ} \mathrm{C}$ for a maximum of 8 hours. ${ }^{63}$ Therefore, continuous infusion of meropenem $3 \mathrm{~g}$ /day could be divided into six consecutive 4-hour continuous infusions of meropenem 0.5 $\mathrm{g}$ in consideration of meropenem stability. Nafcillin sodium was stable for at least 7 days at $25^{\circ} \mathrm{C}$ after reconstitution in $0.9 \%$ sodium chloride injection and storage in polypropylene syringes, which could support the application of continuous infusion (12 hours) of nafcillin. ${ }^{64}$

\section{Infusion rate-related medication administration errors}

It is important to make sure that the fluid will be infused at the prescribed rate. Adverse events can occur if a large continuous infusion dose is administered via accidental rapid infusion. An observational study showed that errors in infusion rates accounted for $10.9 \%$ of drug-related problems associated with intravenous medication administration. ${ }^{65} \mathrm{~A}$ questionnaire survey showed that the wrong infusion rate $(33.3 \%)$ was the most prevalent type of medication errors of nurses in the emergency department. ${ }^{66}$

\section{Further opportunities}

There are many further opportunities in research and clinical practice with respect to optimal infusion rate in antimicrobial therapy. First, it is necessary to conduct head-to-head comparative studies of infusion regimens with different infusion rates from multiple perspectives (eg, clinical outcomes, resources utilization, nurse satisfaction, cost-effectiveness).

Second, dosing and administration strategies should be individualized on the basis of the pathogen-drug-patient interplay to optimize outcomes. For time-dependent antimicrobials, the factors affecting the indice $\% \mathrm{~T}>\mathrm{MIC}$ or $\% \mathrm{fT}>\mathrm{MIC}$ (eg, dose, dosing frequency, infusion rate, MIC value, protein binding, clearance alternations) may have an influence on the difference between two infusion methods. In this review, the factors determining whether prolonged or continuous infusion has a benefit over standard infusion are summarized in Table 2. Therefore, subgroup analysis in specific circumstances and populations should be encouraged, and their findings may provide explanations for the inconsistency of conclusions derived from outcome comparison between extended infusion and standard infusion of antimicrobials. Also, there may be a very pronounced difference in $\mathrm{PK} / \mathrm{PD}$ of antibiotics in neonates and adults; therefore, the conclusion drawn from adult studies could not be simply extrapolated to neonates or pediatric patients.

Third, implementing effective interventions is required to enhance the application of optimal infusion strategies. A survey of Australia and New Zealand ICU doctors and pharmacists showed that a greater proportion of pharmacists compared with doctors believed continuous infusion to be more effective than intermittent administration $(85.4 \%$ vs $34.3 \%$, respectively; $P<0.001) .{ }^{67}$ The pharmacy and therapeutics committee should draft an institutional protocol for the optimal infusion rate of some drugs and implement extensive quality assurance monitoring (eg, retrospective analysis of guideline compliance). ${ }^{68}$ Key elements contributing to the successful implementation of a piperacillin/ tazobactam extended-infusion guideline included consistent pharmacy leadership, multidisciplinary involvement, thorough inservicing to health care professionals, hospital-wide implementation, and extensive quality assurance monitoring. ${ }^{69}$ Sanford Medical Center successfully achieved a great total cost savings of about 2 million US dollar to the hospital (ie, reduction in piperacillin/tazobactam expenditures and additional cost savings due to reduced LOS by 0.6 days) in the first 2 years through automatic conversion from a 30-minute infusion to a 4-hour extended infusion of piperacillin/tazobactam along with staff education and use of smart pump technology. ${ }^{70}$

Fourth, prescribing information on the infusion rate of some medications should be revised according to the updated evidence. Clinicians may not dare to prescribe and administer antimicrobial agents with new infusion strategies due to potential medical disputes and hidden dangers caused by off-label use.

\section{Conclusion}

To maximally preserve the effectiveness of current antimicrobials and prevent further antimicrobial resistance, effective interventions should be implemented to enhance the application of optimal infusion strategies. For reducing nephrotoxicity, prolonged infusion of meropenem over 4 hours is better than the conventional infusion strategy (30 minutes) for meningitis and Pseudomonas infection in neonates with Gram-negative late-onset sepsis; and continuous infusion of vancomycin is superior to intermittent infusion. For increasing drug efficacy, prolonged or continuous infusion of time-dependent antibiotics (eg, meropenem, doripenem, imipenem, cefepime, ceftazidime, piperacillin/tazobactam, linezolid, and vancomycin) is an optimal choice. Extended infusion of piperacillin/tazobactam has pharmacoeconomic 
Table 2 The factors determining whether prolonged or continuous infusion has a benefit over standard infusion

\begin{tabular}{|c|c|}
\hline Factors & Descriptions on whether prolonged or continuous infusion has a benefit over standard infusion \\
\hline $\begin{array}{l}\text { MIC of } \\
\text { bacterial } \\
\text { pathogens }\end{array}$ & $\begin{array}{l}\text { Meropenem: The PTA (ie, } 40 \% \text { T>MIC) with short ( } 0.5 \text {-hour) infusion of I g meropenem every } 8 \text { hours was higher than those } \\
\text { with prolonged (3-hour) infusion regimens for CPKP isolates with MIC } 4-8 \mathrm{mg} / \mathrm{L} \text { rather than MIC } \geq 16 \mathrm{mg} / \mathrm{L} \text { or } \leq 2 \mathrm{mg} / \mathrm{L} \text { (MIC } 4 \\
\mathrm{mg} / \mathrm{L} \text { : } 98 \%-99 \% \text { vs } 61 \%-83 \% \text {; MIC } 8 \mathrm{mg} / \mathrm{L}: 55 \%-79 \% \text { vs } 23 \%-33 \%) . .^{14} \\
\text { Piperacillin/tazobactam: The benefits of prolonged infusion were selective and most likely observed in patients with less } \\
\text { susceptible pathogens, ie, prolonged infusion had no advantages over standard infusion against isolates with susceptible MICs of } 8 \\
\text { or } 16 \mathrm{mg} / \mathrm{L} \text {, whereas it produced more than twice the final bacterial kill against less susceptible isolates with an intermediate MIC } \\
\text { of } 32 \mathrm{mg} / \mathrm{L} .^{39} \\
\text { Piperacillin/tazobactam: For pathogens with MICs of } \leq 8 \mathrm{mg} / \mathrm{L} \text {, extended infusion did not substantially improve PTA, and standard } \\
\text { infusion was likely sufficient. However, piperacillin/tazobactam I00/I } 2.5 \mathrm{mg} / \mathrm{kg} \text { given as an extended infusion every } 6-8 \text { hours } \\
\text { may be optimal for empirical or directed therapy in critically ill pediatric patients with infections caused by less susceptible } \\
\text { pathogens. }{ }^{40}\end{array}$ \\
\hline $\begin{array}{l}\text { Diagnosis } \\
\text { and } \\
\text { disease }\end{array}$ & $\begin{array}{l}\text { Piperacillin/tazobactam: Patients with severe disease (SOFA score } \geq 9) \text {, rather than those with mild disease (SOFA score }<9 \text { ), } \\
\text { benefited from prolonged infusion (eg, shorter days of antibiotics use and ventilator time, longer survival, better clinical efficacy, } \\
\text { and lower } 28 \text {-day mortality rate). }{ }^{41}\end{array}$ \\
\hline severity & $\begin{array}{l}\text { Piperacillin/tazobactam: ICU patients exhibited no significant differences in outcomes following two dosing methods (extended } \\
\text { infusion over } 4 \text { hours vs regular infusion over } 30 \text { minutes), whereas patients with urinary or intra-abdominal infections had lower } \\
\text { mortality and clinical failure rates following extended infusion treatment. }{ }^{42} \\
\text { Piperacillin/tazobactam: There was a lower mortality rate in the extended-infusion group who had infectious organisms identified } \\
\left(9.3 \% \text { vs } 22.4 \%, P=0.0 \text { I) or were diagnosed with respiratory tract infections }(8.9 \% \text { vs } 18.7 \%, P=0.02){ }^{43}\right. \\
\text { Meropenem: ICU patients exhibited the lowest target attainment rates, whereas internal medicine patients achieved the highest } \\
\text { target attainment rates following short ( } 0.5 \text {-hour) and prolonged (3-hour) infusion regimens of I g meropenem every } 8 \text { hours. }{ }^{14}\end{array}$ \\
\hline $\begin{array}{l}\text { Bacterial } \\
\text { density }\end{array}$ & $\begin{array}{l}\text { Piperacillin/tazobactam: for the lower initial bacterial density, trough total plasma piperacillin concentration/MIC ratios of } 3.4 \\
\text { and } 10.4 \text { following bolus and extended-infusion regimens, respectively, were able to suppress the emergence of piperacillin } \\
\text { resistance. For the higher initial bacterial density, both infusion regimens were associated with progressive growth of a resistant } \\
\text { subpopulation, and thus combination therapy may be required to maximize bacterial killing and prevent antimicrobial resistance. }{ }^{44}\end{array}$ \\
\hline $\begin{array}{l}\text { Total } \\
\text { daily dose }\end{array}$ & $\begin{array}{l}\text { Imipenem/cilastatin: In critically ill patients with nosocomial pneumonia, concentrations of imipenem were above the target } \\
\text { concentration ( } 4 \times \mathrm{MIC} \text { of } 2 \mathrm{mg} / \mathrm{L}) \text { for } \geq 40 \% \text { of the dosing interval in every patient in the bolus group ( } \mathrm{g} \text { imipenem/I } \mathrm{g} \text { cilastatin } \\
\text { over } 30 \text { minutes every } 8 \text { hours), whereas in the extended group ( } 0.5 \mathrm{~g} \text { of imipenem by a } 3 \text {-hour infusion every } 6 \text { hours) this } \mathrm{PK} / \\
\mathrm{PD} \text { index ( } 40 \% \mathrm{fT}>4 \times \mathrm{MIC}) \text { was achieved in only } 20 \% \text { of patients. Moreover, } 70 \% \text { of the patients in the extended group did not } \\
\text { reach the desired drug concentrations at all. }{ }^{22}\end{array}$ \\
\hline $\begin{array}{l}\text { Renal } \\
\text { clearance }\end{array}$ & $\begin{array}{l}\text { Meropenem: In adult patients with septic shock and possible increased renal clearance, meropenem doses should be increased } \\
\text { and/or administration by prolonged or continuous infusion should be considered to increase the likelihood of obtaining } \\
\text { therapeutic drug concentrations. In patients with normal renal function, however, standard intermittent dosing ( } 30 \text { minutes) } \\
\text { seems to be sufficient. }{ }^{15}\end{array}$ \\
\hline
\end{tabular}

Abbreviations: CPKP, carbapenemase-producing Klebsiella pneumoniae; ICU, intensive care unit; MIC, minimum inhibition concentration; PK/PD, pharmacokinetic/ pharmacodynamic; PTA, probability of target attainment; SOFA, sequential organ failure assessment; T>MIC, time for which drug concentrations exceed the MIC.

advantage over standard infusion. Nevertheless, the advantage of prolonged or continuous infusion vs regular infusion of time-dependent antibiotics may only exhibit in special clinical circumstance and special populations (eg, patients with SOFA score $\geq 9$, respiratory tract infections, urinary or intra-abdominal infections, or infections caused by less susceptible pathogens, benefited from prolonged infusion of piperacillin/tazobactam).

\section{Acknowledgments}

This work was supported by Zhejiang Provincial Bureau of Education (grant nos. N20140209 and Y201635841). Also, we would like to thank Professor Xuan-ding Wang (Dean of the Department of Hospital Infection Management), Mr JunShou (inpatient pharmacy supervisor), and Yang-min $\mathrm{Hu}$ (clinical pharmacist) for their kind participation in quality improvements in antimicrobial therapy.

\section{Disclosure}

The authors report no conflicts of interest in this work.

\section{References}

1. Joint Commission Resources. Joint Commission International accreditation standards for hospitals. 6th ed. Oak Brook, IL: Joint Commission Resources; 2017.

2. Versporten A, Zarb P, Caniaux I, et al. Antimicrobial consumption and resistance in adult hospital inpatients in 53 countries: results of an internet-based global point prevalence survey. Lancet Glob Health. 2018;6(6):e619-e629.

3. Guervil DJ, Chau T. Trends in multidrug-resistant gram-negative bacilli and the role of prolonged $\beta$-lactam infusion in the intensive care unit. Crit Care Nurs Q. 2013;36(4):345-355. 
4. Lutsar I, Telling K, Metsvaht T. Treatment option for sepsis in children in the era of antibiotic resistance. Expert Rev Anti Infect Ther. 2014;12(10):1237-1252.

5. Roberts JA, Abdul-Aziz MH, Davis JS, et al. Continuous versus intermittent $\beta$-lactam infusion in severe sepsis. A meta-analysis of individual patient data from randomized trials. Am J Respir Crit Care Med. 2016;194(6):681-691.

6. Teo J, Liew Y, Lee W, Kwa AL. Prolonged infusion versus intermittent boluses of $\beta$-lactam antibiotics for treatment of acute infections: a meta-analysis. Int J Antimicrob Agents. 2014;43(5):403-411.

7. Arnold HM, Hollands JM, Skrupky LP, et al. Prolonged infusion antibiotics for suspected gram-negative infections in the ICU: a before-after study. Ann Pharmacother. 2013;47(2):170-180.

8. Dulhunty JM, Roberts JA, Davis JS, et al. A multicenter randomized trial of continuous versus intermittent $\beta$-lactam infusion in severe sepsis. Am J Respir Crit Care Med. 2015;192(11):1298-1305.

9. Abdul-Aziz MH, Sulaiman H, Mat-Nor MB, et al. Beta-Lactam Infusion in Severe Sepsis (BLISS): a prospective, two-centre, open-labelled randomised controlled trial of continuous versus intermittent beta-lactam infusion in critically ill patients with severe sepsis. Intensive Care Med. 2016;42(10):1535-1545.

10. Shabaan AE, Nour I, Elsayed Eldegla H, Nasef N, Shouman B, AbdelHady H. Conventional versus prolonged infusion of meropenem in neonates with gram-negative late-onset sepsis: a randomized controlled trial. Pediatr Infect Dis J. 2017;36(4):358-363.

11. Fehér C, Rovira M, Soriano A, et al. Effect of meropenem administration in extended infusion on the clinical outcome of febrile neutropenia: a retrospective observational study. J Antimicrob Chemother. 2014;69(9):2556-2562.

12. Zhao HY, Gu J, Lyu J, et al. Pharmacokinetic and pharmacodynamic efficacies of continuous versus intermittent administration of meropenem in patients with severe sepsis and septic shock: a prospective randomized pilot study. Chin Med J. 2017;130(10):1139-1145.

13. Mathew SK, Mathew BS, Neely MN, et al. A nonparametric pharmacokinetic approach to determine the optimal dosing regimen for 30-minute and 3-hour meropenem infusions in critically ill patients. Ther Drug Monit. 2016;38(5):593-599.

14. Vourli S, Tsala M, Kotsakis S, et al. Comparison of short versus prolonged infusion of standard dose of meropenem against carbapenemase-producing Klebsiella pneumoniae isolates in different patient groups: a pharmacokinetic-pharmacodynamic approach. J Pharm Sci. 2016;105(4):1513-1518.

15. Sjövall F, Alobaid AS, Wallis SC, Perner A, Lipman J, Roberts JA. Maximally effective dosing regimens of meropenem in patients with septic shock. J Antimicrob Chemother. 2018;73(1):191-198.

16. Abdul-Aziz MH, Lipman J, Akova M, et al. Is prolonged infusion of piperacillin/tazobactam and meropenem in critically ill patients associated with improved pharmacokinetic/pharmacodynamic and patient outcomes? An observation from the defining antibiotic levels in intensive care unit patients (DALI) cohort. JAntimicrob Chemother. 2016;71(1):196-207.

17. Pettit RS, Neu N, Cies JJ, et al. Population pharmacokinetics of meropenem administered as a prolonged infusion in children with cystic fibrosis. J Antimicrob Chemother. 2016;71(1):189-195.

18. Langan KM, Jacob J, Li J, et al. Pharmacokinetics of short versus extended infusion meropenem dosing in critically ill patients: a pilot study. Crit Care Resusc. 2014;16(3):190-196.

19. Hsaiky L, Murray KP, Kokoska L, Desai N, Cha R. Standard versus prolonged doripenem infusion for treatment of gram-negative infections. Ann Pharmacother. 2013;47(7-8):999-1006.

20. Ibrahim MM, Tammam TF, Ebaed MED, Sarhan HA, Gad GF, Hussein AK. Extended infusion versus intermittent infusion of imipenem in the treatment of ventilator-associated pneumonia. Drug Des Devel Ther. 2017;11:2677-2682.

21. Suchánková H, Lipš M, Urbánek K, Neely MN, Strojil J. Is continuous infusion of imipenem always the best choice? Int J Antimicrob Agents. 2017;49(3):348-354.
22. Lipš M, Siller M, Strojil J, Urbánek K, Balík M, Suchánková H. Pharmacokinetics of imipenem in critically ill patients during empirical treatment of nosocomial pneumonia: a comparison of $0.5-\mathrm{h}$ and 3-h infusions. Int J Antimicrob Agents. 2014;44(4):358-362.

23. Dong J, Xiong W, Chen Y, et al. Optimal dosing regimen of biapenem in Chinese patients with lower respiratory tract infections based on population pharmacokinetic/pharmacodynamic modelling and Monte Carlo simulation. Int J Antimicrob Agents. 2016;47(3):202-209.

24. Wiskirchen DE, Housman ST, Quintiliani R, Nicolau DP, Kuti JL. Comparative pharmacokinetics, pharmacodynamics, and tolerability of ertapenem $1 \mathrm{gram} /$ day administered as a rapid 5-minute infusion versus the standard 30-minute infusion in healthy adult volunteers. Pharmacotherapy. 2013;33(3):266-274.

25. Bauer KA, West JE, O'Brien JM, Goff DA. Extended-infusion cefepime reduces mortality in patients with Pseudomonas aeruginosa infections. Antimicrob Agents Chemother. 2013;57(7):2907-2912.

26. Wrenn RH, Cluck D, Kennedy L, Ohl C, Williamson JC. Extended infusion compared to standard infusion cefepime as empiric treatment of febrile neutropenia. J Oncol Pharm Pract. 2018;24(3):170-175.

27. Huang H, Huang S, Zhu P, Xi X. Continuous versus intermittent infusion of cefepime in neurosurgical patients with post-operative intracranial infections. Int J Antimicrob Agents. 2014;43(1):68-72.

28. Cousson J, Floch T, Guillard T, et al. Lung concentrations of ceftazidime administered by continuous versus intermittent infusion in patients with ventilator-associated pneumonia. Antimicrob Agents Chemother. 2015;59(4):1905-1909.

29. Chan JD, Dellit TH, Lynch JB. Hospital length of stay among patients receiving intermittent versus prolonged piperacillin/tazobactam infusion in the intensive care units. J Intensive Care Med. 2018;33(2):134-141.

30. Winstead EM, Ratliff PD, Hickson RP, Mueller JE, Judd WR. Evaluation of an alternative extended-infusion piperacillin-tazobactam dosing strategy for the treatment of gram-negative infections. Int J Clin Pharm. 2016;38(5):1087-1093.

31. Shotwell MS, Nesbitt R, Madonia PN, et al. Pharmacokinetics and pharmacodynamics of extended infusion versus short infusion piperacillintazobactam in critically ill patients undergoing CRRT. Clin J Am Soc Nephrol. 2016;11(8):1377-1383.

32. Jamal JA, Roberts DM, Udy AA, et al. Pharmacokinetics of piperacillin in critically ill patients receiving continuous venovenous haemofiltration: a randomised controlled trial of continuous infusion versus intermittent bolus administration. Int J Antimicrob Agents. 2015;46(1):39-44.

33. McCormick H, Tomaka N, Baggett S, et al. Comparison of acute renal injury associated with intermittent and extended infusion piperacillin/ tazobactam. Am J Health Syst Pharm. 2015;72(11 Suppl 1):S25-S30.

34. Mousavi M, Zapolskaya T, Scipione MR, Louie E, Papadopoulos J, Dubrovskaya Y. Comparison of rates of nephrotoxicity associated with vancomycin in combination with piperacillin-tazobactam administered as an extended versus standard infusion. Pharmacotherapy. 2017;37(3):379-385.

35. Brunetti L, Poustchi S, Cunningham D, et al. Clinical and economic impact of empirical extended-infusion piperacillin-tazobactam in a community medical center. Ann Pharmacother. 2015;49(7):754-760.

36. Schmees PM, Bergman SJ, Strader BD, Metzke ME, Pointer S, Valenti KM. Outcomes of an extended-infusion piperacillin-tazobactam protocol implementation in a community teaching hospital adult intensive care unit. Am J Health Syst Pharm. 2016;73(11 Suppl 3):S100-S105.

37. Bao H, Lv Y, Wang D, Xue J, Yan Z. Clinical outcomes of extended versus intermittent administration of piperacillin/tazobactam for the treatment of hospital-acquired pneumonia: a randomized controlled trial. Eur J Clin Microbiol Infect Dis. 2017;36(3):459-466.

38. Cotrina-Luque J, Gil-Navarro MV, Acosta-García H, et al. Continuous versus intermittent piperacillin/tazobactam infusion in infection due to or suspected pseudomonas aeruginosa. Int J Clin Pharm. 2016;38(1):70-79.

39. Zelenitsky S, Nash J, Weber Z, Iacovides H, Ariano R. Targeted benefits of prolonged-infusion piperacillin-tazobactam in an in vitro infection model of Pseudomonas aeruginosa. J Chemother. 2016;28(5):390-394. 
40. Nichols K, Chung EK, Knoderer CA, et al. Population pharmacokinetics and pharmacodynamics of extended-infusion piperacillin and tazobactam in critically ill children. Antimicrob Agents Chemother. 2016;60(1):522-531.

41. Lyu Y, Yang Y, Li X, et al. Selection of piperacillin/tazobactam infusion mode guided by SOFA score in cancer patients with hospital-acquired pneumonia: a randomized controlled study. Ther Clin Risk Manag. 2017;14:31-37.

42. Cutro SR, Holzman R, Dubrovskaya Y, et al. Extended-Infusion versus standard-infusion piperacillin-tazobactam for sepsis syndromes at a tertiary medical center. Antimicrob Agents Chemother. 2014;58(8):4470-4475.

43. Fan SY, Shum HP, Cheng WY, Chan YH, Leung SM, Yan WW. Clinical outcomes of extended versus intermittent infusion of piperacillin/ tazobactam in critically ill patients: a prospective clinical trial. Pharmacotherapy. 2017;37(1):109-119.

44. Felton TW, Goodwin J, O'Connor L, et al. Impact of bolus dosing versus continuous infusion of Piperacillin and Tazobactam on the development of antimicrobial resistance in Pseudomonas aeruginosa. Antimicrob Agents Chemother. 2013;57(12):5811-5819.

45. Bauer KA, Gentene AJ, West JE, Shidham G, Goff DA. An antimicrobial stewardship program's evaluation of the safety and efficacy of continuous infusion of nafcillin in the treatment of methicillin-sensitive staphylococcus aureus bacteremia. Infect Dis Clin Pract. 2013;21(2):111-113.

46. Cai Y, Bai N, Liu X, Liang B, Wang J, Wang R. Pharmacokinetic/ pharmacodynamic research on three different infusion time regimens of linezolid in healthy Chinese volunteers. Int J Clin Pharmacol Ther. 2015;53(9):765-771.

47. De Pascale G, Fortuna S, Tumbarello M, et al. Linezolid plasma and intrapulmonary concentrations in critically ill obese patients with ventilator-associated pneumonia: intermittent vs continuous administration. Intensive Care Med. 2015;41(1):103-110.

48. Tafelski S, Nachtigall I, Troeger U, et al. Observational clinical study on the effects of different dosing regimens on vancomycin target levels in critically ill patients: continuous versus intermittent application. $J$ Infect Public Health. 2015;8(4):355-363.

49. van Maarseveen EM, Gipmans S, Vasbinder E, Petjak M, van Zanten AR. Switching from intermittent to continuous infusion of vancomycin in critically ill patients: toward a more robust exposure. Ther Drug Monit. 2016;38(3):398-401.

50. Hong LT, Goolsby TA, Sherman DS, et al. Continuous infusion vs intermittent vancomycin in neurosurgical intensive care unit patients. $J$ Crit Care. 2015;30(5):e1-e6.

51. Lin H, Bukovskaya Y, de Moya M, Lee J, Schmidt U. Vancomycin continuous infusion versus intermittent infusion during continuous venovenous hemofiltration: slow and steady may win the race. Ann Intensive Care. 2015;5:10.

52. Schmelzer TM, Christmas AB, Norton HJ, Heniford BT, Sing RF. Vancomycin intermittent dosing versus continuous infusion for treatment of ventilator-associated pneumonia in trauma patients. Am Surg. 2013;79(11):1185-1190.

53. Hanrahan T, Whitehouse T, Lipman J, Roberts JA. Vancomycinassociated nephrotoxicity: A meta-analysis of administration by continuous versus intermittent infusion. Int $J$ Antimicrob Agents. 2015;46(3):249-253.
54. Hao JJ, Chen H, Zhou JX. Continuous versus intermittent infusion of vancomycin in adult patients: a systematic review and meta-analysis. Int J Antimicrob Agents. 2016;47(1):28-35.

55. Wu Y, Kang J, Wang Q. Drug concentrations in the serum and cerebrospinal fluid of patients treated with norvancomycin after craniotomy. Eur J Clin Microbiol Infect Dis. 2017;36(2):305-311.

56. Albiero J, Sy SK, Mazucheli J, et al. Pharmacodynamic evaluation of the potential clinical utility of fosfomycin and meropenem in combination therapy against KPC-2-producing Klebsiella pneumoniae. Antimicrob Agents Chemother. 2016;60(7):4128-4139.

57. Hohmann N, Kreuter R, Blank A, et al. Autoinhibitory properties of the parent but not of the N-oxide metabolite contribute to infusion rate-dependent voriconazole pharmacokinetics. Br J Clin Pharmacol. 2017;83(9):1954-1965.

58. Moriyama B, Kadri S, Henning SA, Danner RL, Walsh TJ, Penzak SR. Therapeutic drug monitoring and genotypic screening in the clinical use of voriconazole. Curr Fungal Infect Rep. 2015;9(2):74-87.

59. Raverdy V, Ampe E, Hecq JD, Tulkens PM. Stability and compatibility of vancomycin for administration by continuous infusion. JAntimicrob Chemother. 2013;68(5):1179-1182.

60. Tran A, O'Sullivan D, Krawczynski M. Cefepime intravenous push versus intravenous piggyback on time to administration of first-dose vancomycin in the emergency department. J Pharm Pract. 2017;897190017734442.

61. Kidd JM, Avery LM, Asempa TE, Nicolau DP, Kuti JL. Physical compatibility of meropenem and vaborbactam with select intravenous drugs during simulated Y-site administration. Clin Ther. 2018;40(2):261-269.

62. Franceschi L, Cojutti P, Baraldo M, Pea F. Stability of generic meropenem solutions for administration by continuous infusion at normal and elevated temperatures. Ther Drug Monit. 2014;36(5):674-676.

63. Carlier M, Stove V, Verstraete AG, de Waele JJ. Stability of generic brands of meropenem reconstituted in isotonic saline. Minerva Anestesiol. 2015;81(3):283-287.

64. Ling J, Gupta VD. Stability of nafcillin sodium after reconstitution in $0.9 \%$ sodium chloride injection and storage in polypropylene syringes for pediatric use. Int J Pharm Compd. 2000;4(6):480-481.

65. Vijayakumar A, Sharon EV, Teena J, Nobil S, Nazeer I. A clinical study on drug-related problems associated with intravenous drug administration. J Basic Clin Pharm. 2014;5(2):49-53.

66. Ehsani SR, Cheraghi MA, Nejati A, Salari A, Esmaeilpoor AH, Nejad EM. Medication errors of nurses in the emergency department. $J$ Med Ethics Hist Med. 2013;6:11.

67. Cotta MO, Dulhunty JM, Roberts JA, Myburgh J, Lipman J. Should $\beta$-lactam antibiotics be administered by continuous infusion in critically ill patients? A survey of Australia and New Zealand intensive care unit doctors and pharmacists. Int J Antimicrob Agents. 2016;47(6):436-438.

68. Hohlfelder B, Kubiak DW, Degrado JR, Reardon DP, Szumita PM Implementation of a prolonged infusion guideline for time-dependent antimicrobial agents at a tertiary academic medical center. Am J Ther. 2016;23(6):e1768-e1773.

69. Heinrich LS, Tokumaru S, Clark NM, Garofalo J, Paek JL, Grim SA. Development and implementation of a piperacillin-tazobactam extended infusion guideline. J Pharm Pract. 2011;24(6):571-576.

70. Maddox ML, DeBoer EC, Hammerquist RJ. Administration of extended infusion piperacillin-tazobactam with the use of smart pump technology. Hosp Pharm. 2014;49(5):444-448.
Infection and Drug Resistance

\section{Publish your work in this journal}

Infection and Drug Resistance is an international, peer-reviewed openaccess journal that focuses on the optimal treatment of infection (bacterial, fungal and viral) and the development and institution of preventive strategies to minimize the development and spread of resistance. The journal is specifically concerned with the epidemiology of antibiotic resistance and the mechanisms of resistance development and diffusion in both hospitals and the community. The manuscript management system is completely online and includes a very quick and fair peerreview system, which is all easy to use. Visit http://www.dovepress.com/ testimonials.php to read real quotes from published authors. 